\title{
Trends in Oncology: an Updated Review of Minimally Invasive Surgery vs. Traditional Open Surgery
}

\author{
Kyuwon Lee, Ahromi Wang \\ Not affiliated with any institution, Seoul, South Korea
}

\section{CORRESPONDENCE}

\section{Ahromi Wang}

595-1 Ahn Kook Building \#202

Sinsa Dong, Gangnam Gu, Seoul,

South Korea

Tel: +82 01046566760

E-mail: ahromi.w@gmail.com

\section{ARTICLE HISTORY}

Received: October 3, 2020

Accepted: October 26, 2020

\begin{abstract}
Objective: To review the recent trends in methodology for the most frequent oncological surgeries. More specifically, this paper will compare the minimally invasive methods to the conventional open method. It aims to find out whether minimally invasive surgeries are feasible treatments for certain types of cancers. Methods: A review of retrospective studies searched in PubMed, Cochrane Library etc. was used to identify the studies published on this topic within a period of 6 years. Oncologic outcomes, postoperative complications/outcomes, and intraoperative outcomes were the main variables in the comparison to the surgical methods under review. Conclusion: Despite the recent controversy with minimally invasive surgery being contraindicated for cervical cancer, it is still an acceptable method for other types of common cancers. However, there are limitations to the approach, and the surgeon should make a prudent selection between minimally invasive and open surgery depending on the type of tumor and patient characteristics. Further studies, especially with randomized control trials, must be conducted.
\end{abstract}

Keywords: surgery, minimally invasive, oncology, review

In recent years, there has been controversy over whether or not minimally invasive surgery (MIS) should remain the gold standard for many surgical procedures, in particular, for radical hysterectomies. The issue was brought to light by two studies in The New England Journal of Medicine, which established that minimally invasive radical hysterectomy was associated with a lower diseasefree survival rate than open surgery. ${ }^{1,2}$ For a number of years, the National Comprehensive Cancer Network ( $\mathrm{NCCN}$ ) guidelines have stated that robotic radical hysterectomies were the recommended approach for patients with cervical cancer, citing the benefits of such an approach. ${ }^{3}$ However, it seems that with the shocking revelation of the dangers of minimally invasive hysterectomies, surgeons should reevaluate their methodology of choice for other surgical procedures as well. Thus, in accordance with what may be a rapid and prema- 
ture adoption of robotics in the field of medicine, an updated review is needed to evaluate the efficacy of MIS in the treatment of other oncological diseases and determine what should be the gold standard method.

\section{Foundational Information on MIS}

MIS emerged in the 1980s to create a safer and more effective form of surgery compared to traditional open surgery. It was designed to meet the surgical needs of many patients. MIS can commonly be separated into three forms: laparoscopic surgery, endoscopic surgery, and robotic surgery. ${ }^{4}$ Laparoscopic surgery is performed through one or more small incisions, using small tubes, tiny cameras, and surgical instruments. Endoscopic surgery is a diagnostic and therapeutic procedure performed through the body's organs and vessels. Robotic surgery is a magnified, $3 \mathrm{D}$ view of the surgical sites to help the surgeon operate with precision, flexibility, and control. It has recently become quite prevalent as it resulted in smaller incisions and shorter hospital stay. It has also been attributed to lower risk compared to that of traditional open surgery, with a reduction of operative traumatic damage.

\section{Public View on MIS}

From the surgeons' perspective, MIS is less preferred than open surgery. However, that may be different from the public view of MIS. As John Wickham stated in 1987, "Surgeons applaud large incisions and denigrate keyhole surgery. Patients, in contrast, want the smallest wound possible, and we at Britain's first department of minimally invasive surgery are convinced that patients are right." 5 The patients definitely prefer MIS as it usually results in shorter hospital stay, smaller incision, and smaller scars. According to a study by Haws et al., patients are very heavily swayed by long-term outcomes, surgeons' recommendations, and risk of complications for both open surgery and MIS. The majority of patients were convinced that open surgeries are more painful (83.8\%), have an increased risk of complications $(78.5 \%)$, increased recovery time $(89.3 \%)$, and increased costs $(68.1 \%) .6$ Overall, especially for spinal surgery, MIS is perceived by patients as a more suitable and better option than open surgery.

\section{Methodology}

The sources that we used to compile our data were The Cochrane Library, Pubmed, The New England Journal of Medicine, and PLOS ONE. We compiled a list of the most frequent operating room procedures and the most common types of cancers, and from the overlap, we determined the final list of surgeries for this study. Then, we compared search results for "open surgery vs. laparoscopic surgery" and found the top results (must have had more than 5 results in the last 5 years). The search terms used were: "open," "laparoscopic," "minimally invasive," and "surgery."

We included papers based on meta-analyses in our review. Some papers were based on a compilation of data from a number of relevant studies. Other papers were based on empirical data or direct case studies.

The main factors that were considered in determining which surgical method was more feasible were: intraoperative outcomes, oncologic safety, postoperative outcomes, and postoperative recovery. Miscellaneous factors were also considered if they were deemed to be significant to the studies under review. We have omitted some studies that were either too outdated (before 2014) or questionable in their accuracy (based on individual case studies). Study results derived from an insufficient number of study groups were omitted for a more accurate and precise analysis. Results comparing laparoscopic vs. robotic surgery with no open control were omitted. Cholecystectomy was also omitted because there were not enough oncological results.

\section{ANALYSIS OF MIS VS. OPEN SURGERY IN DIFFERENT TYPES OF CANCERS}

\section{Hepatectomy}

Compared to laparoscopic gastrectomies, there are more studies available for laparoscopic hepatectomies. This is because laparoscopic liver resection was already widespread decades ago. Thus, the laparoscopic approach for liver resection is not a novel procedure but rather part of the standard guidelines for surgery.

In 2014, the 2nd International Consensus on Laparoscopic Liver Resection had constituted MIS to be the standard method of treatment (IDEAL 3) for low-risk or minor hepatectomy. However, they defined MIS for major hepatectomies (resection of more than 3 segments) to still be innovative (IDEAL 2b). There were not enough highquality studies conducted to push MIS to be the standard method for all types of hepatectomies. ${ }^{7}$

Three years later, 2,819 patients participated in a large, multi-institutional study that compared MIS to the open approach for major hepatectomies. ${ }^{8}$ This study was significant because it was the largest one yet to collect data on 
minimally invasive major liver surgeries with an open control. Although the result did not immediately confer absolute confidence to the advocates of MIS for high-risk surgeries, it did show a sort of optimism for the approach. The conclusion was that MIS was not worse than open surgery for patients with large tumors (one-sided $95 \% \mathrm{CI}<1.02$ ).

Not long after that, the first randomized controlled study had been conducted comparing laparoscopic and open liver resection. ${ }^{9}$ The trial included 280 patients with resectable liver metastases and found that the post-operative complication rate was significantly lower for the laparoscopic group (19\% vs. $31 \%$ ). The study is in definitive support of laparoscopic liver surgery and is evidence enough that it should be continued to be implemented in the field. Furthermore, a recent compendium of case studies $(n=233)$ showed the success of major laparoscopic resection. ${ }^{10}$

In conclusion, it is generally agreed that minor hepatectomies should be performed laparoscopically. In addition, while the approach for major hepatectomies should be determined based on the skill of the surgeon, the difficulty of the surgery, and patient condition, it could very well prove to be the more favored method in the near future.

\section{Pancreaticoduodenectomy}

A 2016 study conducted by Zhang et al. compared laparoscopic pancreaticoduodenectomy (LPD) and open pancreaticoduodenectomy (OPD). ${ }^{11}$ The study examined whether LPD (a subset of minimally invasive PD) is as safe and feasible as OPD, assessing the improvement in R0 resection after LPD. PD has always been a complex procedure due to the dissection around important vessels and three complex reconstructions. The major difference between the two PDs were significantly reduced blood loss and blood transfusion requirement with LPD compared to OPD, and a shorter postoperative stay (6 days vs. 9 days).

However, there are some studies that refute the possibility of LPD leading to a faster rate of R0 resection, and therefore, it was concluded that LPD cannot be considered a significantly "better" procedure than OPD. It was also suggested that there is a learning curve for LPD. As a consequence, higher morbidity and mortality were associated with LPD at the beginning of the learning curve. ${ }^{12}$ Nevertheless, given the clear-cut benefits of less blood loss and shorter postoperative stays, LPD proves to be a safer and more feasible method for patients.

The same study also looked at the feasibility of pancreaticoduodenectomy, compiling all studies conducted between January 1994 and November 2013. During the compilation of the evidence to assess the benefits of laparoscopic procedures, the authors looked for wound infection, length of operation time, intraoperative blood loss, risk of complications, length of hospital stays, and overall oncologic outcomes. The study determined that there was a reduction in wound infection due to the minimally invasive approach, but the laparoscopic procedures have taken longer during operation because of their complexity and the lack of experience of surgeons. Some of the difficulties in pancreaticoduodenectomy were partly due to the difficulty in access and exposure of the pancreas, difficulty in hemorrhage control from major vasculature, and difficulty in reconstruction of biliary and pancreatic remnants. However, procedure complexity has largely been alleviated by the development of surgical robotics. This advancement led to significantly shorter operation times for minimally invasive pancreaticoduodenectomy (MIPD): $444 \pm 93.5$ minutes vs. $559 \pm 135$ minutes with OPD. The study also determined that there was a significant reduction in blood loss, lower risk of complications, shorter hospital stay, and overall better oncologic outcomes. It concluded that due to its advantages, MIPD is worthwhile. The complexity of MIPD has been alleviated and will continue to become better through advancements in surgical robotics and more experienced surgeons in the future. ${ }^{12}$

\section{Esophagectomy}

Two studies were carried out to determine whether minimally invasive esophagectomy (MIE), or even hybrid minimally invasive esophagectomy (HMIE), should replace open procedures for esophageal cancer. The first study determined that HMIE and MIE yielded shorter hospital stays due to the lower number of excised lymph nodes. ${ }^{13}$ There were also lower rates of pulmonary complications and anastomotic leaks, as well as better outcomes in the MIE groups in certain situations. However, MIE had longer operation times, and survival benefits were similar in OE and MIE. In addition, there was a longer learning curve for MIE due to the highly advanced laparo-thoracoscopic skills required. The first study concluded that although there are clear benefits to MIE and HMIE over OE, longer learning curves and longer operating times due to procedure complexity of the procedure make it hard for minimally invasive surgery to be used more widely. For esophageal cancer, MIE and HMIE should be used only in high-volume, experienced university surgical centers. Another study based on 13,267 patients who had esophageal cancer determined that there were similar short- and 
long-term survival rates between hybrid, minimal, and open procedures. ${ }^{14}$ The study demonstrated that in-hospital mortality was reduced in MIE (3\% for MIE vs. $4.6 \%$ for open surgery), and the risk of pulmonary complications was reduced in MIE (17.8\% for MIE vs. $20.4 \%$ for open). However, there wasn't an apparent reduction of morbidity or mortality in the MIE group compared with the open esophagectomy group. In addition, there was no significant difference in the rate of anastomotic leaks after esophagectomy between the two groups.

\section{Adrenalectomy}

Regarding adrenalectomies, MIS is the general method of choice. As of 2013, laparoscopic adrenalectomy has been recommended for benign adrenal tumors ( $\leq 6 \mathrm{~cm}$ in diameter and weighing $<100 \mathrm{~g}$ ). For tumors greater than $6 \mathrm{~cm}$, there is an ongoing debate between endocrinologists regarding the surgical method. In a study conducted by Conzo et al. in 2015, when laparoscopic adrenalectomy (LA) and open adrenalectomy (OA) were performed for lesions $>10 \mathrm{~cm}$, the recurrence rate was $50 \%$ and $42 \%$, respectively. ${ }^{15} \mathrm{LA}$ was contraindicated in the presence of intravenous thrombus and infiltration of structures. It was noted that an increased risk of peritoneal carcinomatosis after LA was a concern; however, results regarding that specific postoperative risk were inconclusive.

But in 2018, another study showed that the laparoscopic approach was acceptable regardless of tumor size and without any associated risk increase. ${ }^{16}$ The study followed 28 patients with 31 adrenal tumors. Tumor sizes were categorized into $<5 \mathrm{~cm}$ and $\geq 5 \mathrm{~cm}$ in diameter. No difference for mortality and recurrence rate between LA and OA was found.

Furthermore, in a 2017 study investigating lateral retroperitoneoscopic adrenalectomy (LRA) versus OA $(n=67$, all tumors were greater than $5 \mathrm{~cm}$; 41 patients by LRA and 26 patients by $\mathrm{OA}$ ), the risk of local recurrence and peritoneal carcinomatosis, a highly associated risk, were found to be comparable for the two approaches. ${ }^{17}$

Taking all above studies into consideration, the interpretation is that for larger adrenal tumors, LA is acceptable but technically demanding, as it has been observed that LA requires longer surgery time. However, this review focuses not on intraoperative factors but postoperative results, so the length of surgery time will be considered negligible when evaluating feasibility. Thus, the relevant issue is which minimally invasive approach, laparoscopic anterior versus endoscopic posterior, will produce better results.

\section{Gastrectomy}

Gastric surgery is one of the most relevant fields for MIS but there are no international guidelines set in place that dictate when and for what type of disease it is indicated. The current gold standard for gastric surgery is the open method. Currently, there are several relevant, ongoing multicenter trials, and results are pending. Unfortunately, data availability along with updated clinical standards have not kept up with the technical advances in the field.

In a case-control study comparing robotic gastrectomy (RG), laparoscopic gastrectomy (LG), and open gastrectomy (OG), ${ }^{18}$ intraoperative blood loss was shown to be significantly lower in the LG $(95.93 \pm 119.22 \mathrm{~mL})$ and RG $(117.91 \pm 68.11 \mathrm{~mL})$ groups compared to the OG $(127.26 \pm$ $79.50 \mathrm{~mL}, \mathrm{p}=0.002)$. The length of hospital stay was shorter for LG and RG, and there was no difference in complication rates regardless of the tumor type. It has shown that the main benefit of MIS is a significantly faster post-surgical recovery rate. A 2017 study has evaluated the effects of laparoscopic distal gastrectomy (LDG) and found that there were fewer pulmonary complications. The mortality rate was similar between the two groups ( $0.4 \%$ for LDG vs. $0 \%$ for ODG, $\mathrm{p}=0.249$ ), and the 5-year survival rate was also similar (95.9\% for LDG vs. $94.9 \%$ for ODG). ${ }^{19}$

For early gastric cancer, LDG is comparable to OG. The general consensus across all recent studies is that there is not enough data to conclusively state the feasibility and safety of LDG for advanced gastric cancer. Studies are still ongoing (particularly to confirm the benefit of the laparoscopic method in elderly patients with advanced gastric cancer), and there is a lack of high-level clinical evidence. ${ }^{20,21}$ Currently, a randomized controlled trial studying the efficacy of laparoscopic subtotal gastrectomy for advanced gastric cancer is being carried out in Korea. ${ }^{22}$ Totally laparoscopic complete gastrectomy (TLCG) has been performed successfully for advanced gastric cancer proving its feasibility, but it faces the steep wall of being technically challenging. Furthermore, oncological results have yet to be recorded..$^{23}$ Laparoscopic gastrectomy continues to evolve due to its touted benefits, technological improvements, and increasing experience of surgeons. Although there are multiple studies regarding the minimally invasive routes, further studies are needed to evaluate the oncological safety of these procedures.

\section{Colorectal Cancer}

One of the studies regarding the trends of minimally invasive surgical resection for colorectal cancer was con- 
ducted on May 16, 2019, based on The Florida Inpatient Discharge Data Set. The study examined the clinical data of patients who underwent elective surgery for colorectal cancer between 2013 and 2015. A total of 10,513 patients were analyzed, of which 5,451 had open surgery, 4,403 had laparoscopic surgery, and 659 had robotic surgery. The rate of MIS increased from $46.95 \%$ to $48.72 \%$ in the study period, and among a variety of minimally invasive procedures, the use of robotics increased from $9.82 \%$ in 2013 to $15.48 \%$ in $2015 .^{24}$ This upward trend in MIS for colorectal cancer shows the popularity of MIS among the general public. Another study, a meta-analysis, explored the overall effect and safety of anterior laparoscopic surgery versus conventional open surgery for patients with colorectal cancer based on 24 randomized controlled trials, with 4,592 patients in the laparoscopic group and 3,865 patients in the open surgery group. Laparoscopic surgery had significantly less blood loss, shorter hospital stay, and lower rates of postoperative mortality and postoperative complications. However, one benefit of open surgery was that laparoscopic surgery had statistically higher operative times than open surgery. The study concluded that despite the shorter operative times for open surgery, laparoscopic surgery was far superior for colorectal cancer due to its major benefits. ${ }^{25}$

Another study aimed to determine the feasibility of conducting laparoscopic colorectal resection in elderly patients, as very old patients with colorectal cancer are at a high risk of mortality. This study compiled previous studies from PubMed, Medline, Embase, and Google Scholar and examined primary determiners, such as 30-day mortality rates, 30-day morbidity rates, and secondary determiners, such as operating time, time to oral diet, number of retrieved lymph nodes, blood loss, and 5-year survival rates. Elective laparoscopic resection had a mortality rate of $2.92 \%$ and a morbidity rate of $23 \%$. The study found significant differences between laparoscopy and open surgery, demonstrating reduced morbidity in the laparoscopic group. In addition, the study determined that laparoscopic surgery patients were more likely to have shorter hospital stays and shorter time to oral diet. The authors concluded that laparoscopic surgery was safer and more feasible than open surgery for patients over the age of $85 .^{26}$

Patients with liver cirrhosis are also at a high risk for colorectal surgery because the safety and effectiveness of laparoscopy in colorectal surgery involving cirrhotic patients is not clear. A study performed in 2018 concluded that laparoscopic colorectal surgery is a safer and less invasive alternative to open surgery in some cirrhotic patients, leading to less blood loss, earlier recovery, and less additional harm in terms of postoperative complications or long-term oncological outcomes. ${ }^{27}$

\section{Lung Cancer}

There is no dispute that lung cancer is a leading cause of death in the world, and the management of the disease remains one of the most important topics in the medical field. The minimally invasive method for lung cancer surgery is termed video-assisted thoracoscopic surgery (VATS), and it has already been established as being just as effective as open surgery. Currently, there is no large dispute on this matter, and there have been multiple studies worldwide (e.g., Sweden, Taiwan, United States) proving the adequacy of VATS. ${ }^{28-30}$ Long-term survival for both minimally invasive and open surgery was equal, while postoperative complications for the former were less frequent.

According to a 2019 study, there has been a significant increase in the number of VATS performed for non-smallcell lung carcinoma (NSCLC) in the U.S. (study limitations: patients were all $\geq 65$ years of age, the number of VATS may have increased secondarily to an increase in the number of diagnoses/resections). ${ }^{31}$ There was a $39 \%$ increase from 2006 to 2014 in VATS carried out in patients who had elected to undergo surgery. However, there was a significant regional variation regarding the management of the disease. Unlike in the case of other cancers where the type of tumor indicated the course of treatment, the staging of the lung cancer was not a factor in the variation of treatment (VATS vs. open). In response to this discrepancy, a number of medical practitioners are advocating for VATS to become the official standard method of treatment so that it can be adopted universally in all hospitals.

\section{Thyroid Cancer}

Head and neck surgeries are usually the most technically demanding interventions because of the general anatomy of the tumors' location. Because thyroid cancer has a high incidence in women, surgeons also consider the cosmetic outcome when performing the procedures. Hence, more surgeons are choosing the minimally invasive approach. However, it requires a specific skill set, may also have higher risks associated with it, and it remains controversial.

One of the most recent meta-analyses comparing endoscopic thyroidectomy (ET) and open thyroidectomy (OT) showed that the two methods were comparable in terms of post-surgical results. ${ }^{32}$ The study took into account some of the most common complications for thyroidectomy such as damage to the recurrent laryngeal nerve and post- 
operative hypoparathyroidism; transient recurrent laryngeal nerve palsy occurred more frequently in ET. Also, the study showed that completeness of thyroid resection may be lower in case of ET because patients were reported to have comparatively higher serum thyroglobulin (sTg) levels. However, there was not enough postoperative data to make a conclusion about sTg levels because there was no significant difference between ET and OT when it came to tumor recurrence rates.

All studies comparing other types of endoscopic thyroidectomies, such as minimally invasive video assisted thyroidectomy (MIVAT) and robotic thyroidectomy, with the conventional method reached the conclusion that ET was comparable to OT. ${ }^{33-36}$ Other studies have shown that not only was MIVAT as safe as open surgery, it may also have beneficial immunosuppressive effects through the downregulation of TNF- $\alpha .{ }^{37}$ Also, there are new risks posed by robotic surgery brachial plexus neuropathy and tracheal injury. ${ }^{38}$

For the minimally invasive method, whether it is MIVAT or robotic surgery, there is a strict learning curve which prevents the application of more innovative methods in a wider setting. Endoscopic methodologies and techniques represent one of the prominent current topics in endocrinology. For the time being, there are limitations set in place for ET, and for higher risk patients OT is the preferred method, but hopefully this will change with continued interest and further studies.

\section{Prostate Cancer}

As prostate cancer is one of the most common types of cancer in men, it has long been debated whether prostatectomy should be performed through laparoscopic or open procedures. A randomized, controlled study of men recently diagnosed with localized prostate cancer examined the functional and oncological postoperative outcomes up to 24 months after laparoscopic or open surgery. The study included 308 men aged between 35 and 70 years, 151 of which were treated with open radical retropubic prostatectomy, while 157 were treated with robot-assisted laparoscopic prostatectomy. The study did not find significant differences between open and laparoscopic surgery regarding urinary and sexual function, the proportion of biochemical recurrences, or the proportion of patients who had imaging evidence of progression. The authors concluded that laparoscopic surgery and open surgery yielded similar functional outcomes at 24 months. The benefits of a robotic approach to prostate cancer would largely be related to its minimally invasive nature. ${ }^{39}$
Another study assessed the effects of laparoscopic radical prostatectomy or robotic-assisted radical prostatectomy compared to open radical prostatectomy in 446 randomized participants with clinically localized prostate cancer. The authors examined primary outcomes, such as prostate cancer-specific survival, urinary quality of life, and sexual quality of life, and secondary outcomes such as biochemical recurrence-free survival, overall survival, overall surgical complications, serious postoperative surgical complications, postoperative pain, hospital stay, and blood transfusion. The study determined that there was no difference between laparoscopic surgery and open surgery in urinary and sexual quality of life, and little to no difference in overall surgical complications and serious postoperative complications. The study also concluded that laparoscopic and robotic procedures are very slightly better in terms of postoperative pain at one day and up to one week. However, there was no significant difference in postoperative pain at 12 weeks. It was also determined that robotic procedures may reduce the length of hospital stay, and both robotic and laparoscopic procedures would result in 68 fewer blood transfusions per 1,000 men compared to open procedures. The study concluded that there was no clear evidence that laparoscopic and robotic procedures would have better oncological outcomes that the open counterpart. The differences between the procedures were minimal to non-existent, all of which were not persuasive evidence to the superiority of laparoscopic procedures. Taking into account the possibility of the surgeon's lack of experience in minimally invasive surgery, as of right now, prostate cancer should be treated through open procedures. ${ }^{40}$

\section{DISCUSSIONS AND CONCLUSIONS}

Reviewing the results of the relevant studies from the past five years, we found that there was an overwhelming amount of data that emphasizes the efficacy of MIS. In many areas, the endoscopic or robotic method has already been adopted as the standard for low-risk surgeries. With the exception of cervical cancer, prostate cancer, and disseminated cancers, there is a definite accelerating trend towards MIS compared to a decade ago.

MIS is safer and more feasible for appendectomies. As for pancreaticoduodenectomy, there are clear benefits that make MIE a better option for most patients. However, there is a need for further research on the long-term outcomes of MIPD. There is a very long learning curve for esophagectomies due to the complexity of the procedure. The studies conducted on esophagectomies require fur- 
ther improvements in surgical devices and surgeon experience. MIS is the method of choice for adrenalectomies, but is restricted to certain tumor sizes. Further studies are needed regarding gastrectomies, and open surgery continues to be the recommended method for prostate cancer. For all other oncological surgeries, MIS is accepted as a feasible treatment but is restricted by the type and grade of the disease.

For the most part, MIS is only comparable/not superior to open surgery, meaning that postoperative complication rates, tumor recurrence rates, and other factors were not significantly different. It was rare to find a paper that reviewed MIS to be the better method of choice without the addendum that further studies were needed to assess long-term risks. Also, if the cancer had progressed to a stage where the tumors were large or disseminated, MIS was contraindicated because resection would not be as complete as with open surgery. The limitations of MIS are also based upon the skills of the surgeon and availability of instruments. Even when it is considered feasible, the surgeon must consider the various endoscopic approaches from different anatomical sites; this remains a topic of controversy and requires further study.

In conclusion, an increasing number of surgeons are choosing the minimally invasive method, and the current trends show that this approach is largely accepted in the medical community.

\section{CONFLICT OF INTEREST}

Nothing to disclose.

\section{REFERENCES}

1. Ramirez PT, Frumovitz M, Pareja R, et al. Minimally Invasive versus Abdominal Radical Hysterectomy for Cervical Cancer. N Engl J Med. 2018;379:1895-1904.

2. Melamed A, Margul DJ, Chen L, et al. Survival after Minimally Invasive Radical Hysterectomy for Early-Stage Cervical Cancer. N Engl J Med. 2018:379:1905-1914

3. National Comprehensive Cancer Network. NCCN. http://www.nccn.org/ about/news/ebulletin/ebulletindetail.aspx?ebulletinid=1569 (09 September 2020)

4. Minimally invasive surgery. Mayo Clinic. Mayo Foundation for Medical Education and Research; 2019. http://www.mayoclinic.org/testsprocedures/minimally-invasive-surgery/about/pac-20384771 (09 September 2020)

5. Schlich T, Tang CL. Patient choice and the history of minimally invasive surgery. The Lancet. 2016;388:1369-1370.

6. Narain AS, Hiji FY, Duhancioglu G, et al. Patient Perceptions of Minimally Invasive Versus Open Spine Surgery. Clin Spine Surg. 2018;31:E184-E192.

7. Wakabayashi G, Cherqui D, Geller DA, et al. Recommendations for laparoscopic liver resection: a report from the second international consensus conference held in Morioka. Ann Surg. 2015;261:619-629.

8. Thornblade LW, Shi X, Ruiz A, Flum DR, Park JO. Comparative Effectiveness of Minimally Invasive Surgery and Conventional Approaches for Major or Challenging Hepatectomy. J Am Coll Surg. 2017;224:851-861.

9. Fretland ÅA, Dagenborg VJ, Bjørnelv GMW, et al. Laparoscopic Versus
Open Resection for Colorectal Liver Metastases: The OSLO-COMET Randomized Controlled Trial. Ann Surg. 2018;267:199-207.

10. Cho W, Kwon CHD, Choi JY, et al. Impact of technical innovation on surgical outcome of laparoscopic major liver resection: 10 years' experience at a large-volume center. Ann Surg Treat Res. 2019;96:14-18.

11. Zhang YH, Zhang CW, Hu ZM, Hong DF. Pancreatic cancer: Open or minimally invasive surgery? World J Gastroenterol. 2016;22:7301-7310

12. Qin H, Qiu J, Zhao Y, Pan G, Zeng Y. Does minimally-invasive pancreaticoduodenectomy have advantages over its open method? A meta-analysis of retrospective studies. PLOS One. 2014;9:e104274.

13. Wallner G, Zgodziński W, Masiak-Segit W, Skoczylas T, Dąbrowski A. Minimally invasive surgery for esophageal cancer - benefits and controversies. Kardiochir Torakochirurgia Pol. 2014;11:151-155.

14. Zhou C, Zhang L, Wang $H$, et al. Superiority of Minimally Invasive Oesophagectomy in Reducing In-Hospital Mortality of Patients with Resectable Oesophageal Cancer: A Meta-Analysis. PLoS One. 2015;10(7):e0132889.

15. Conzo G, Pasquali D, Della Pietra C, et al. Laparoscopic adrenal surgery: ten-year experience in a single institution. BMC Surg. 2013;13Suppl2:S5.

16. Mohammed A, Amine H, Atiq SE, et al. Applicability and outcome of laparoscopic adrenalectomy for large tumours. Pan Afr Med J. 2018;31:23.

17. Chen W, Lin W, Han DJ, Liang Y. Lateral retroperitoneoscopic adrenalectomy for complicated adrenal tumor larger than 5 centimeters. Afr Health Sci. 2017;17:293-300.

18. Parisi A, Reim D, Borghi F, et al. Minimally invasive surgery for gastric cancer: A comparison between robotic, laparoscopic and open surgery. World J Gastroenterol. 2017;23:2376-2384.

19. Hu Y, Huang C, Sun Y, et al. Morbidity and Mortality of Laparoscopic Versus Open D2 Distal Gastrectomy for Advanced Gastric Cancer: A Randomized Controlled Trial. J Clin Oncol. 2016;34:1350-1357.

20. Li Z, Shan F, Ying X, Xue K, Ji J. Laparoscopic versus open gastrectomy for elderly local advanced gastric cancer patients: study protocol of a phase I randomized controlled trial. BMC Cancer. 2018;18:1118.

21. Son T, Hyung WJ. Laparoscopic gastric cancer surgery: Current evidence and future perspectives. World J Gastroenterol. 2016;22:727-735.

22. Hur H, Lee HY, Lee HJ, et al. Efficacy of laparoscopic subtotal gastrectomy with D2 lymphadenectomy for locally advanced gastric cancer: the protocol of the KLASS-02 multicenter randomized controlled clinical trial. BMC Cancer. 2015;15:355.

23. Shinohara T, Hanyu N, Tanaka Y, Murakami K, Watanabe A, Yanaga K. Totally laparoscopic complete resection of the remnant stomach for gastric cancer. Langenbecks Arch Surg. 2013;398:341-345.

24. Osagiede O, Spaulding AC, Cochuyt JJ, Naessens J, Merchea A, Colibaseanu DT. Trends in the Use of Laparoscopy and Robotics for Colorectal Cancer in Florida. J Laparoendosc Adv Surg Tech A. 2019:29:926-933

25. Song XJ, Liu ZL, Zeng R, Ye W, Liu CW. A meta-analysis of laparoscopic surgery versus conventional open surgery in the treatment of colorectal cancer. Medicine (Baltimore). 2019;98:e15347.

26. Devoto L, Celentano V, Cohen R, Khan J, Chand M. Colorectal cancer surgery in the very elderly patient: a systematic review of laparoscopic versus open colorectal resection. Int J Colorectal Dis. 2017;32:1237-1242.

27. Zhou S, Zhu H, Li Z, Ying X, Xu M. Safety of laparoscopic resection for colorectal cancer in patients with liver cirrhosis: A retrospective cohort study. Int J Surg. 2018;55:110-116.

28. Al-Ameri M, Bergman P, Franco-Cereceda A, Sartipy U. Video-assisted thoracoscopic versus open thoracotomy lobectomy: a Swedish nationwide cohort study. J Thorac Dis. 2018;10:3499-3506.

29. Wang BY, Huang JY, Lin CH, et al. Thoracoscopic Lobectomy Produces Long-Term Survival Similar to That with Open Lobectomy in Cases of Non-Small Cell Lung Carcinoma: A Propensity-Matched Analysis Using a Population-Based Cancer Registry. J Thorac Oncol. 2016:11:1326-1334.

30. Ezer N, Kale M, Sigel K, et al. Outcomes after Video-assisted Thoracoscopic Lobectomy versus Open Lobectomy for Early-Stage Lung Cancer in Older Adults. Ann Am Thorac Soc. 2018;15:76-82.

31. Phillips JD, Bostock IC, Hasson RM, et al. National practice trends for the surgical management of lung cancer in the CMS population: an atlas of care. J Thorac Dis. 2019;11(Supp|4):S500-S508.

32. Chen C, Huang S, Huang A, et al. Total endoscopic thyroidectomy versus conventional open thyroidectomy in thyroid cancer: a systematic review and meta-analysis. Ther Clin Risk Manag. 2018;14:2349-2361.

33. Zhang P, Zhang HW, Han XD, Di JZ, Zheng Q. Meta-Analysis of Comparison Between Minimally Invasive Video-Assisted Thyroidectomy and Conventional Thyroidectomy. European Review of Medical and Pharmacological Sciences. 2015;19:1381-1387. 
34. Li $Y$, Zhou $X$. Comparison between endoscopic thyroidectomy and conventional open thyroidectomy for papillary thyroid microcarcinoma: A meta-analysis. J Can Res Ther. 2016;12:550-555.

35. Tan Z, Gu J, Han Q, et al. Comparison of Conventional Open Thyroidectomy and Endoscopic Thyroidectomy via Breast Approach for Papillary Thyroid Carcinoma. Int J Endocrinol. 2015;2015:239610.

36. Cho MJ, Park KS, Cho MJ, Yoo YB, Yang JH. A comparative analysis of endoscopic thyroidectomy versus conventional thyroidectomy in clinically lymph node negative thyroid cancer. Ann Surg Treat Res. 2015;88:69-76.

37. Zheng C, Liu S, Geng P, et al. Minimally invasive video-assisted versus conventional open thyroidectomy on immune response: a meta analysis. Int J Clin Exp Med. 2015;8:2593-2599.
38. Caruso G, Spinosi MC, Cambi J, Passali FM, Bellussi L, Passali D. Open versus robotic thyroidectomy: is it really a controversial choice? Kulak Burun Bogaz Ihtis Derg. 2015;25:375-376.

39. Coughlin GD, Yaxley JW, Chambers SK, et al. Robot-assisted laparoscopic prostatectomy versus open radical retropubic prostatectomy: 24-month outcomes from a randomised controlled study. Lancet Oncol. 2018;19:10511060

40. Ilic D, Evans SM, Allan CA, Jung JH, Murphy D, Frydenberg M. Laparoscopic and robotic-assisted versus open radical prostatectomy for the treatment of localised prostate cancer. Cochrane Database Syst Rev. 2017;9:CD009625. 